\title{
The combined maximum principle in the problem of synthesis of an adaptive dynamic filter under conditions of disturbances in the measurement process
}

\author{
Andrey Kostoglotov ${ }^{1}$, Aleksandr Kuzin ${ }^{1}$, Sergey Lazarenko ${ }^{2}$ and Igor Pugachev ${ }^{2}$, \\ ${ }^{1}$ Rostov State Transport University, 344038, Rostov-on-Don, Russia \\ ${ }^{2}$ Don State Technical University, 344002, Rostov-on-Don, Russia
}

\begin{abstract}
The article considers the problem of estimating the parameters of motion under conditions of disturbances in the measurement process, which are caused by missing data, misses in measurements, etc. in the operation of radar systems. The structure of the included filter for estimating the parameters of motion is determined by the mathematical model of the maneuvering target. At present time the kinematic models are widely used, but they do not fully correspond to the observed dynamics. This may lead to divergence of the estimation process and failure of the computational procedure. New dynamic filters of the combined maximum principle with the dynamic model of motion possess higher accuracy and stability and smaller amount of computational costs in comparison with common filters. The materials and methods We propose new mathematical model that determines the structure of the dynamic filter of the combined maximum principle. A numerical simulation of the operation of the proposed filter, as well as comparison of its efficiency with common filters by a number of criteria are performed. The results. The efficiency of functioning of radio engineering systems on the basis of the solution to the problem of synthesis of a dynamic filter using method of the combined maximum principle with construction of adaptive dynamic model of motion in vector-matrix form is increased. This makes it possible to eliminate the divergence of computational estimation procedures caused by the inadequacy of the structure of the mathematical model of motion. It is shown that the developed dynamic filter differs from the known filters in terms of the structure of the transition matrix. Discussion and conclusions The results of the mathematical modeling showed that in comparison with the Kalman filter and the "alpha-beta" filter the new dynamic filter under conditions of disturbances in the measuring process increase the number of performance indicators.
\end{abstract}

\section{Introduction}

The structure of the filterb for estimating the parameters of motion is determined by the mathematical model of the maneuvering target. Among them kinematic models, describing the simplest variants of motion with a constant speed or acceleration, are widely used [1]. More complex types of trajectories require to use the shaping filter [2]. Estimation of the motion parameters of the observed target implies the need to solve the multidimensional Stratonovich equation, which is quite difficult to perform in real time, so in practice the method of the Gaussian approximation of the probability density is used. The quasi-optimal filter (the extended Kalman filter) is very widely used. In this case, the additive combination of the observed coordinate and the white Gaussian noise with known intensity appears as the observation model. However, the real operating conditions are characterized by disturbances in the measurement process because of data skipping, rough measurement errors, and so on [3]. The kinematic model does not fully correspond to the observed dynamics, for example, in case of maneuvering the target. Then the misses are hidden and not excluded from observations [4]. All these factors lead to the divergence of the estimation process and hence to the failure of the computational procedure [1-4].

Thus, the operating of the filters under conditions of disturbances in measuring processes requires to adapt the filters. This leads to more complicated estimation procedures and to increase in the computational costs, which limits the use of such solutions for estimating multidimensional processes in real time $[1,4]$.

The work is aimed to solve the problem of decreasing of the effectiveness of radar systems under conditions of disturbances in the measurement process due to the divergence or failure of the estimation computational procedures because of the low adequacy of the mathematical model of motion which the filter is based on.

The filters of the combined maximum principle [512] with a dynamic motion model, which is obtained on the basis of the solution to the problem of the control synthesis [12], are distinguished by high accuracy and a

\footnotetext{
"Corresponding author: bakut_8536@mail.ru
} 
small amount of computational costs in comparison with the common filters.

The aim of the research is to increase the efficiency of radar systems under conditions of disturbances in the measurement process based on the solution to the problem of synthesis of an adaptive dynamic filter using the combined maximum principle.

It is shown in [12] that the use of the hypothesis about the stationarity of the Hamiltonian on the switching surface considering the transversality conditions leads to a dynamic motion model. Its application provides the synthesis of the new dynamic filter. Mathematical modeling of the measurement of the target bearing measurement under perturbation conditions confirms its high efficiency in comparison with the Kalman filter and its clones ( $\alpha-\beta$ filter) $[1,2$, 12]. It is found that the application of the new approach provides an increase in the tracking factor, a decrease in the coefficient of false paths and the average lifetime of the false paths with high accuracy and reduction of the computational costs.

\section{Formulation of the problem}

In the observation space we define the target functional $[5,12]$ :

$$
J=\frac{1}{2} \int_{0}^{t_{1}}[\mathbf{y}(t)-\mathbf{q}(t)]^{T} \mathbf{N}^{-1}[\mathbf{y}(t)-\mathbf{q}(t)] d t=\int_{0}^{t_{1}} F(\mathbf{y}, \mathbf{q}, t) d t,(1)
$$

where $\mathbf{N}^{-1}$ is the weight matrix characterizing the intensity of interference in the observation channel

$$
\mathbf{y}(t)=\mathbf{q}(t)+\xi(t),
$$

here $\mathbf{q} \in R^{n}$ is the vector of generalized coordinates, $\xi(t) \in R^{n}$ is the vector of random effects on the observation channel of known intensity, $t \in\left[0, t_{1}\right] \subset R$, $n$ is the number of degrees of freedom of the dynamical system.

The observed dynamics satisfies the HamiltonOstrogradskii principle [11]. Let us write the extended action functional in the form [5-10]

$$
S=\int_{0}^{t_{1}}[\lambda(T+A)+F] d t
$$

where $\quad T=T(\mathbf{q}, \dot{\mathbf{q}}, t)=\frac{1}{2} \dot{\mathbf{q}}^{T} \dot{\mathbf{q}}$ is the kinetic energy, $A=\int_{q(0)}^{q\left(t_{1}\right)} \sum_{s=1}^{n} Q_{s} d q_{s} \quad$ is the work of generalized forces $Q_{s}=Q_{s}(\mathbf{q}, \dot{\mathbf{q}}, t)$ at the true trajectory,

$$
\mathbf{Q} \in \bar{G}
$$

here $\bar{G}$ is the set of generalized forces admitting the observed motion, $\lambda=\lambda(\xi, \mathbf{q}, \dot{\mathbf{q}})$ is the Lagrange multiplier depending on the vector of random effects $\xi(t)$ and on the trajectory $(\mathbf{q}, \dot{\mathbf{q}}) \in R^{2 n}$.

It is required to find the vector of generalized forces $\mathbf{Q}(\mathbf{q}, \dot{\mathbf{q}}, \xi, \lambda)$ as a function of the trajectory $(\mathbf{q}, \dot{\mathbf{q}}) \in R^{2 n}$ and random effects $\xi \in R^{n}$.

The solution to this problem is based on the methodology of the combined maximum principle [512]. To refine the structure of the solution obtained, it is necessary to analyze the behavior of the invariants of motion on the characteristic trajectories in the phase space.

\section{The synthesis of the dynamic filter}

The condition of the minimum of the target functional can be represented in the form of the condition of the maximum of the generalized power [5-12]

$$
\Phi(\mathbf{q}, \dot{\mathbf{q}}, \mathbf{Q}, \lambda, \xi)=\max \sum_{s=1}^{n}\left[\lambda Q_{s}+V_{s}\right] \dot{q}_{s}
$$

where $\quad \mathbf{V}=\operatorname{gradF}$;

under the transversality condition

$$
\left.H\right|_{0} ^{t_{1}}=\lambda(A+T)+\left.F\right|_{0} ^{t_{1}}
$$

Expressions (5) and (6) serve as the basis to buils the quasideterministic motion model [8], which, taking into account the chosen form of kinetic energy, can be reduced to a quasilinear form with respect to the highest derivative $[8,11]$

$$
\ddot{q}_{s}=\left[-\sqrt{\lambda^{-1}} \dot{q}_{s}+\lambda^{-1} N_{s s}^{-1}\left[y_{s}-q_{s}\right]\right], s=\overline{1, n}
$$

The vector-matrix representation (7) leads to the first-order differential equation

$$
\begin{aligned}
& \dot{\widetilde{\mathbf{q}}}=\widetilde{\mathbf{\Phi}} \widetilde{\mathbf{q}}+\mathbf{N}^{-1} \widetilde{\mathbf{H}}^{*}[\mathbf{y}-\widetilde{\mathbf{H}} \widetilde{\mathbf{q}}], \\
& \widetilde{\mathbf{q}}(0)=\widetilde{\mathbf{q}}^{0},
\end{aligned}
$$

where

$$
\begin{gathered}
\widetilde{\mathbf{q}}=\left[\begin{array}{c}
q_{1} \\
\dot{q}_{1} \\
\vdots \\
q_{n} \\
\dot{q}_{n}
\end{array}\right], \widetilde{\mathbf{H}}=\left[\begin{array}{lllll}
1 & 0 & \cdots & 1 & 0
\end{array}\right], \widetilde{\mathbf{H}}^{*}=\left[\begin{array}{c}
0 \\
\lambda^{-1} \\
\vdots \\
0 \\
\lambda^{-1}
\end{array}\right], \\
\widetilde{\mathbf{\Phi}}=\left[\begin{array}{ccccc}
0 & 1 & \cdots & 0 & 0 \\
0 & -\sqrt{\lambda^{-1}} & \cdots & 0 & 0 \\
\vdots & \vdots & \ddots & \vdots & \vdots \\
0 & 0 & \cdots & 0 & 1 \\
0 & 0 & \cdots & 0 & -\sqrt{\lambda^{-1}}
\end{array}\right] .
\end{gathered}
$$




\section{The results}

Let us consider the process of the bearing measurement in the discrete time

$$
y(k)=\mathbf{H x}(k)+\xi(k),
$$

where $\mathbf{x}(k)$ is the extended state vector with structure determined by the mathematical model of motion, $\mathbf{H}$ is the matrix of the projection of the space of states onto the space of observations, $\xi(k)$ is the white Gaussian noise with known spectral density $N, k$ is the current time. 16]

The second order kinematic model has the form $[1$,

$$
\mathbf{x}(k+1)=\mathbf{\Phi}(\Delta T) \mathbf{x}(k)+\mathbf{G} v(k),
$$

where $\boldsymbol{\Phi}(\Delta T)=\left|\begin{array}{cc}1 & \Delta T \\ 0 & 1\end{array}\right|$ is the transition matrix, $\mathbf{x}=\left[\begin{array}{l}x_{1} \\ x_{2}\end{array}\right], x_{1}(k)=q(k \Delta T), x_{2}(k)=\dot{x}_{1}(k)$, are the bearing and the speed of its change respectively, $\mathbf{G}$ is vector of the perturbations intensity, $v$ the exciting function, $\Delta T$ is the data sampling period.

The Kalman filter equations (KF) can be represented by the expression $[1,2]$

$$
\begin{aligned}
& \hat{\mathbf{x}}(k+1 \mid k)=\mathbf{\Phi}(\Delta T) \hat{\mathbf{x}}(k \mid k), \\
& \hat{\mathbf{x}}(k \mid k)=\hat{\mathbf{x}}(k \mid k-1)+\mathbf{K}(k \mid k-1)(y(k)-\mathbf{H} \hat{\mathbf{x}}(k \mid k-1)], \\
& \mathbf{K}(k \mid k-1)=\mathbf{P}(k \mid k-1) \mathbf{H}^{T}\left[\mathbf{H P}(k \mid k-1) \mathbf{H}^{T}+N^{2}\right]^{-1,}, \\
& \mathbf{P}(k \mid k-1)=\mathbf{\Phi}(\Delta T) \mathbf{P}(k-1 \mid k-1) \mathbf{\Phi}^{T}(\Delta T)+\mathbf{V}(k), \\
& \mathbf{P}(k \mid k)=\mathbf{P}(k \mid k-1)-\mathbf{P}(k \mid k-1) \mathbf{H}^{T}\left[\mathbf{H P}(k \mid k-1) \mathbf{H}^{T}+N^{2}\right]^{-1} \mathbf{H P}(k \mid k-1),
\end{aligned}
$$

where $\hat{\mathbf{x}}(k+1 \mid k)$ is the estimation $\mathbf{x}(k+1)$ based on all previous measurements, including the last $k$-th value (one step prediction), $\hat{\mathbf{x}}(k \mid k)$ is the estimation $\mathbf{x}(k)$ based on all previous measurements (filtering), $\mathbf{K}(k \mid k-1)$ is the feedback coefficient, $\mathbf{P}(k \mid k), \mathbf{P}(k \mid k-1)$ are the matrices of estimation and one step prediction respectively,

$$
\mathbf{V}=\left|\begin{array}{ll}
\frac{1}{3} \Delta T^{3} & \frac{1}{2} \Delta T^{2} \\
\frac{1}{2} \Delta T^{2} & \Delta T
\end{array}\right|
$$

is covariant matrix of the perturbations vector $[1,2,16]$; $\mathbf{H}=\left|\begin{array}{ll}1 & 0\end{array}\right|$.

For the mathematical model (10) the expressions for the amplification coefficient and state covariance allow us to go to the $\alpha-\beta$ filter, where the Kalman filter amplification coefficients are determined by the coefficients $\alpha$ and $\beta[1,2,11]$ :

$$
\begin{aligned}
& \hat{\mathbf{x}}(k+1 \mid k)=\boldsymbol{\Phi}(\Delta T) \hat{\mathbf{x}}(k \mid k), \\
& \hat{\mathbf{x}}(k \mid k)=\hat{\mathbf{x}}(k \mid k-1)+\left[\begin{array}{c}
\alpha \\
\frac{\beta}{\Delta T}
\end{array}\right][y(k)-\mathbf{H} \hat{\mathbf{x}}(k \mid k-1)]
\end{aligned}
$$

The use of (8) leads to the new dynamic filter for estimating the parameters of motion in discrete time

$$
\begin{aligned}
& \hat{\mathbf{x}}(k+1)=\boldsymbol{\Phi}(\Delta T) \hat{\mathbf{x}}(k)+\mathbf{H}^{*}[y(k)-\mathbf{H} \hat{\mathbf{x}}(k)], \\
& \mathbf{H}^{*}=\left[\begin{array}{c}
0 \\
\Delta T \lambda^{-1} N^{-1}
\end{array}\right], \boldsymbol{\Phi}(\Delta T)=\left[\begin{array}{cc}
1 & \Delta T \\
0 & 1-\Delta T \sqrt{\lambda^{-1}}
\end{array}\right],
\end{aligned}
$$

where sign $\wedge$ denotes estimation.

The mathematical modeling results are shown in Figures 1-3. The performance indicators of the filters (11), (12), (13) are summarized in the Table 1.

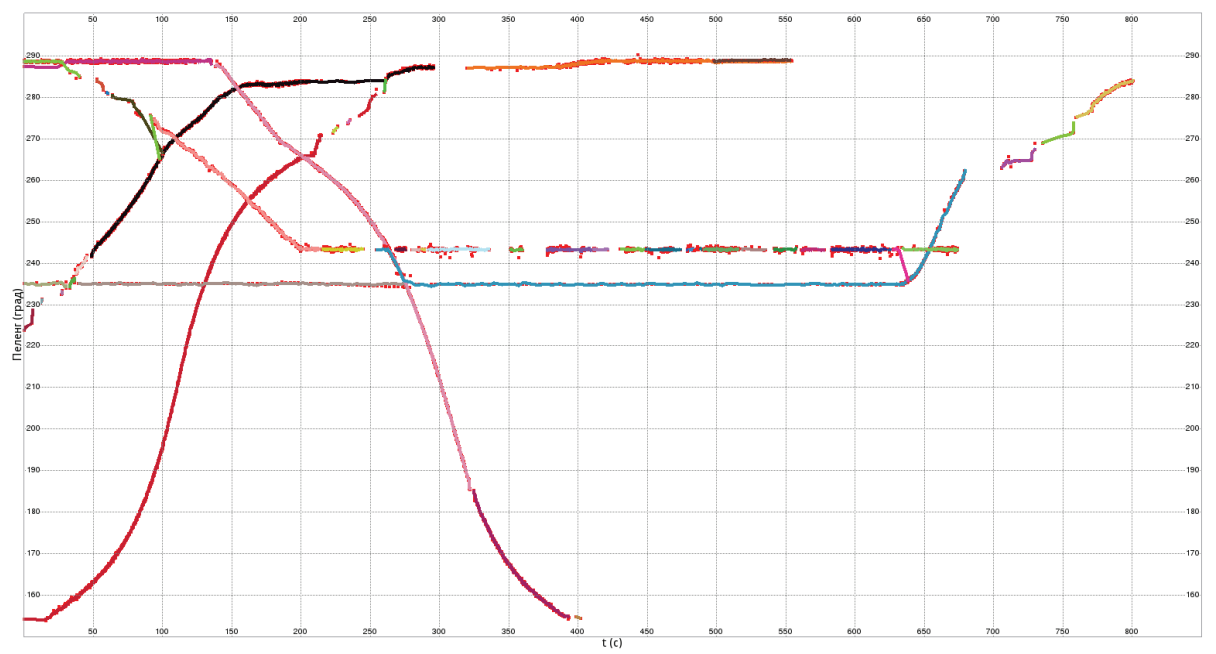

Fig. 1. Bearing estimates by the Kalman filter. 


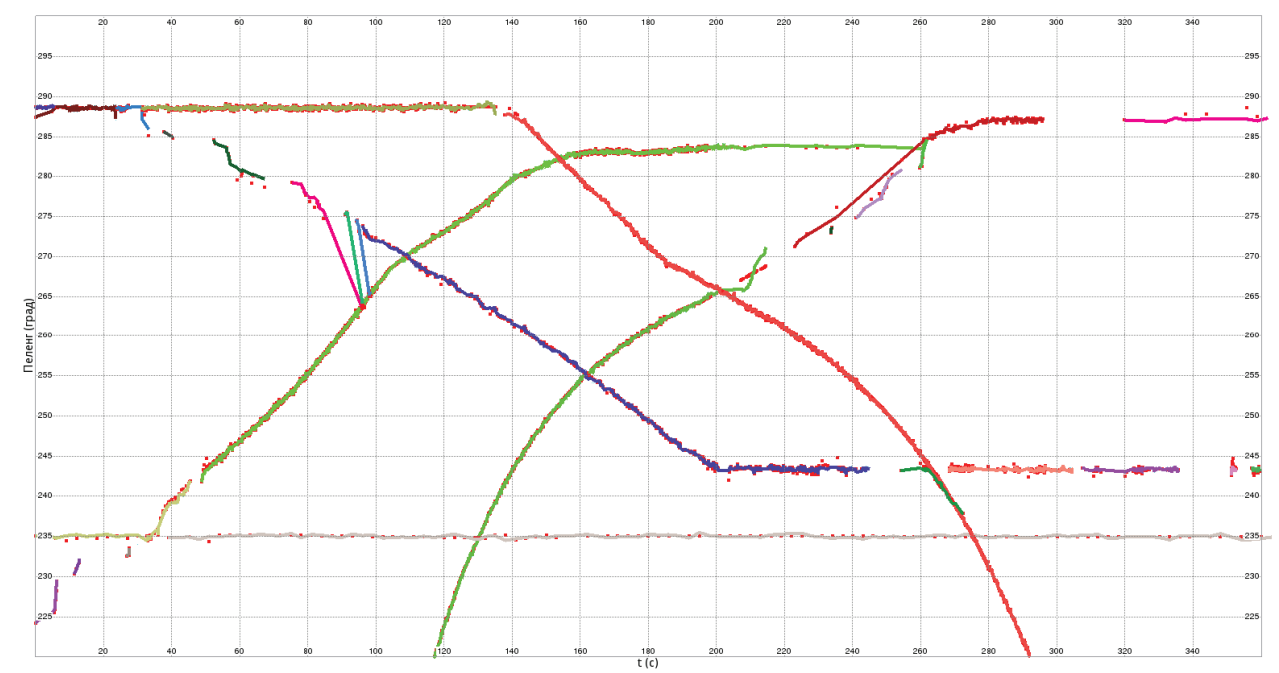

Fig. 2. Bearing estimates by the $\alpha-\beta$ filter.

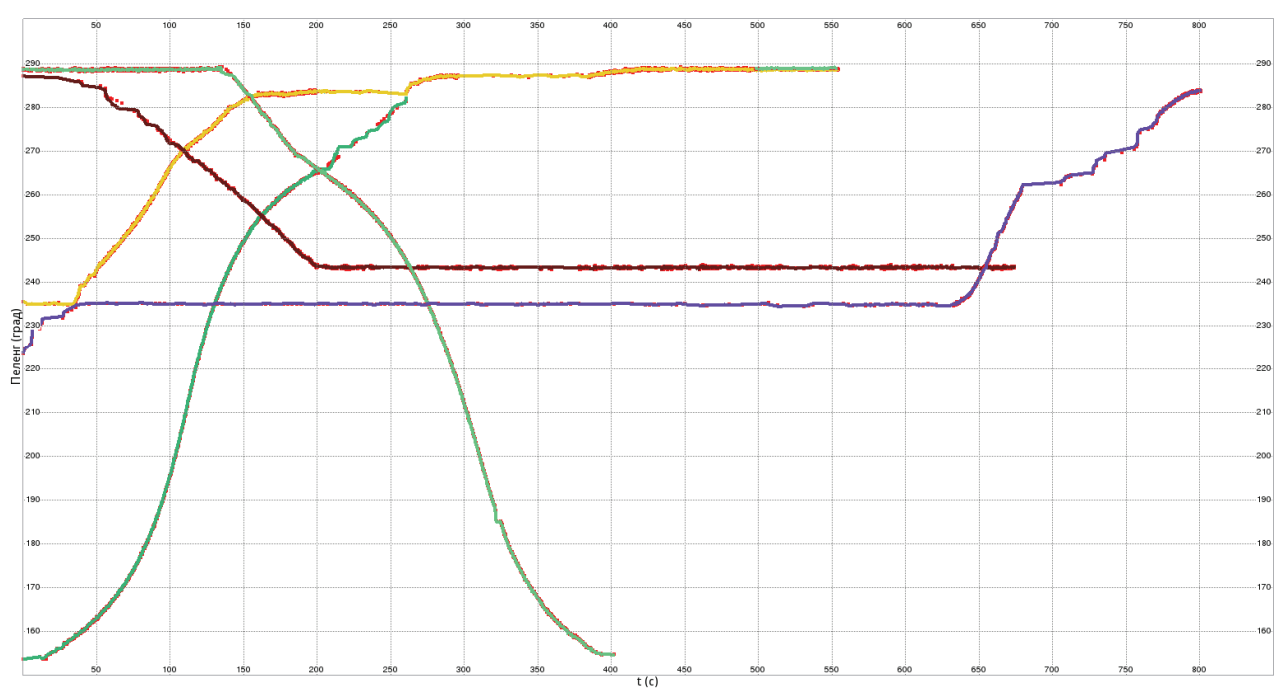

Fig. 3. Bearing estimates by the dynamic filter.

Table 1.Filters Characteristics

\begin{tabular}{|l|l|l|l|l|l|}
\hline $\begin{array}{l}\text { The } \\
\text { performance } \\
\text { indicators }\end{array}$ & $\begin{array}{l}\text { The averaged } \\
\text { estimation error, } \\
\text { deg }\end{array}$ & $\begin{array}{l}\text { The averaged } \\
\text { tracking coefficient }\end{array}$ & $\begin{array}{l}\text { The averaged } \\
\text { coefficient of the } \\
\text { false paths }\end{array}$ & $\begin{array}{l}\text { The averaged } \\
\text { lifetime of the false } \\
\text { paths }\end{array}$ & $\begin{array}{l}\text { The averaged } \\
\text { lifetime of the paths } \\
\text { discontinuity }\end{array}$ \\
\hline Filters & 1.05 & 0.95 & 0.044 & 3 & 8.0 \\
\hline The CMP filter & 1.34 & 0.86 & 0.130 & 15 & 8.4 \\
\hline KF & 1.17 & 0.77 & 0.225 & 8 & 7.2 \\
\hline
\end{tabular}

\section{Discussion and conclusions}

The use of a dynamic motion model as the basis for the new filter provides an increase in the efficiency of its operating under conditions of disturbances in the measuring process with respect to a number of parameters in comparison with the Kalman filter and the "alpha-beta" filter $[1,2,12]$.

The paper has been accomplished with the support of Russian Federal Property Fund grants No. 15-08-03798. 


\section{References}

1. Y. Bar-Shalom, Li X. Rong, T. Kirubarajan. Estimation with Applications to Tracking and Navigation (John Wiley \& Sons, New York, 2001)

2. R.A. Singer and K.W. Behnke, IEEE Transactions on Aerospace and Electronic Systems, no.1, $100-110$ (1971)

3. A.A. Kostoglotov, S.V. Lazarenko, D.S. Andrashitov, and I.V. Deryabkin. Synthesis of Phaselocked Loop System Structure with Adaptation Based on Combined-maximum Principle, available at: $\quad$ http://www.matecconferences.org/articles/matecconf/abs/2016/40/mate cconf icmmr2016 15002/matecconf icmmr2016 15 002.html (accessed 10.09.17).

4. A.A. Kostoglotov, I.V. Deryabkin, S.V. Lazarenko, I.V. Pugachev, and A.A. Kuznetsov. In Proc. EWDTS, 310 - 314 (2016)

5. A.A. Kostoglotov, S.V. Lazarenko. Journal of Communications Technology and Electronics, 121 125 (2017)

6. S.V. Lazarenko, I.V. Pugachev, A.A. Kostoglotov, I.V. Deryabkin, and D.S. Andrashitov. In Proc. ICMSC 2017, 292 - 297 (2017)

7. A.A. Kostoglotov, O.A. Kostoglotova, I.V. Deryabkin, I.E. Kirillov, and S.V. Lazarenko. In Proc. ICMSC 2017, 216 - 220 (2017)

8. A.A. Kostoglotov, I.V. Deryabkin, D.S. Andrashitov, S.V. Lazarenko, and A.A. Kuznetcov. In Proc. ICMSC 2017, 367 - 372 (2017)

9. A.A. Kostoglotov, S.V. Lazarenko, and Z.V. Lyaschenko. In Proc. 2017 20th IEEE International
Conference on Soft Computing and Measurements, SCM 2017, 568 - 571 (2017)

10. C.C. Schooler, IEEE Transactions on Aerospace and Electronic Systems, no 6,1300 - 1306 (1975)

11. A.I.Lur'e. Analytical mechanics (GIFML, Moscow: 1961)

12.X. R. Li, V. P. Jilkov. IEEE Transactions on Aerospace and Electronic Systems, no.4, 1333 1364 (2003) 